\title{
Spin degrees of freedom and flattening of the spectra of single-particle excitations in strongly correlated Fermi systems
}

\author{
V. A. Khodel ${ }^{1}$, P. Schuck ${ }^{2}$, and M. V. Zverev ${ }^{1}$ \\ 1 Kurchatov Institute, Russian Research Center, \\ Moscow, 123182 Russia \\ 2 Groupe de Physique Théorique, \\ Institut de Physique Nucléaire, \\ F-01406 Orsay Cedex, France
}

(Dated: November 17, 2018)

\begin{abstract}
The impact of long-range spin-spin correlations on the structure of a flat portion in single-particle spectra $\xi(p)$, which emerges beyond the point, where the Landau state loses its stability, is studied. We supplement the well-known Nozieres model of a Fermi system with limited scalar long-range forces by a similar long-range spin-dependent term and salculate the snertra versus its strenoth $a$ It is found that Nozieres results hold as long as $g$ spontaneous magnetization is shown to arise at any demonstrated to result in shrinkage of the domain in of $\xi(p)$, and, eventually, in its vanishing.
\end{abstract}

The investigation of flattening of single-particle (sp) spectra of Fermi liquids is dated back to Ref. [1], where long-range correlations, enhanced in the vicinity of an impending ferromagnetic phase transition, were shown to result in the divergence of the effective mass $M^{*}$ at the transition point. Later in 2] an idea of the so-called fermion condensation, i.e. a rearrangement of the Landau state, occurring beyond a critical point in strongly correlated Fermi systems with long-range effective interactions, was suggested. A striking feature of this rearrangement is "swelling" of the Fermi surface (FS), i.e. the occurrence of a completely flat portion $\xi(\mathbf{p})=0$, called the fermion condensate (FC), in the spectrum $\xi(\mathbf{p})$, measured from the FS.

To gain insight into the problem of fermion condensation, let us turn to the Dyson equation, rewriting it in the form

$$
\xi(\mathbf{p})=\xi_{\mathbf{p}}^{0}+\Sigma(\mathbf{p}, \xi(\mathbf{p})),
$$

appropriate for finding the $\mathrm{FC}$ solutions $\xi(\mathbf{p})=0$. The sp mass operator $\Sigma$ is usually determined by the formula

$$
\left.\Sigma(\mathbf{p}, \varepsilon)=\int W \mathbf{p}, \varepsilon, \mathbf{p}_{1}, \varepsilon_{1}\right) G\left(\mathbf{p}_{1}, \varepsilon_{1}\right) \frac{d^{4} p_{1}}{(2 \pi)^{4} i},
$$

where $W$ is an effective interaction between particles, and $G$ is the sp Green function. It is worth noting that the imaginary part of $\Sigma(p, \varepsilon)$ vanishes at $\varepsilon=0$. Therefore, in searching for the FC solutions, only the real part of $\Sigma$ is relevant.

For a long time, it was reckoned that in homogeneous Fermi systems, there exists a one-to-one correspondence between the momentum $p$ and the sp energy $\xi$, at least, close to the FS, i.e. the derivative $(d \xi / d p)_{F}$ is always positive, a postulate, virtually being a cornerstone of the Landau theory of Fermi liquid [3]. However, in systems with long-range forces it might be incorrect. Compelling evidence for that is provided by a phenomenological model, suggested by Nozieres [4], where $W$ is taken as

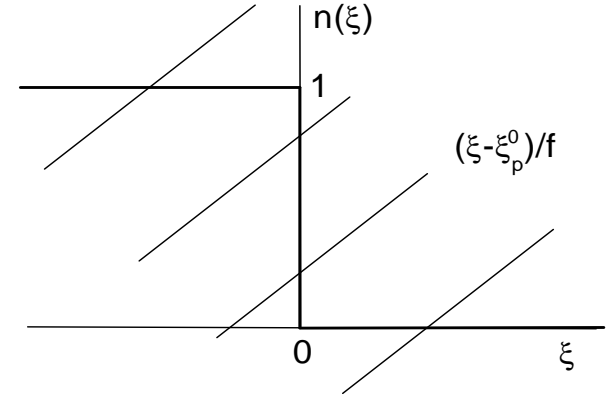

FIG. 1: Graphical illustration of the solution of Eq. (3).

a constant in the coordinate space. Thus, in this model Eq. (11) takes the form

$$
\xi-\xi_{\mathbf{p}}^{0}=f n(\xi),
$$

where $f$ is an effective coupling constant, the value of which is taken to be positive, while $n(\xi)$ is the Landau quasiparticle momentum distribution, being 0 at positive $\xi$, and 1 , at negative ones. This equation is easily solved, but we concentrate on its graphical solution (see Fig. 1). Let us draw both the r.h.s. and the l.h.s. of Eq. (3) as functions of $\xi$, taking $p$ as an input parameter. The l.h.s. of (3), depicted at different $p$, provides a set of parallel straight lines, while the r.h.s. forms a kink, the vertical segment of which is located at $\xi=0$, no matter what is the input. Crossing points yield the sp spectrum $\xi(p)$. If they lie on any of the two horizontal pieces of the kink, $\xi(p)$ does coincide with the sp spectrum of ideal Fermi gas. An unconventional situation occurs when straight lines cross the steep section of the kink. In this case, the intersection point remains 0 in a finite momentum interval $\left[p_{i}, p_{f}\right]$. This plateau $\xi(p)=0$, lying exactly at the FS, does form the fermion condensate. Since $\xi(p)=0$ in the finite volume of momentum space, the density of states $\rho(\varepsilon)$ acquires a singular term $\rho(\varepsilon) \sim \delta(\varepsilon)$. Another salient feature of the fermion condensation phenomenon 
is that in the FC domain, the quasiparticle momentur distribution $n_{0}(p)$ differs from the Landau one. Indeed upon setting $\xi=0$ in Eq.(3i) one finds

$$
n_{0}(p)=-\frac{\xi_{\mathbf{p}}^{0}}{f}, \quad p_{i}<p<p_{f}
$$

in contrast to the conventional step function $n_{F}(p)=$ $\theta\left(p_{F}-p\right)$. Thus, the FS does swell, whereas the basic assertion of Landau theory fails.

In dealing with the FC problem, attention is usually paid to the spin-independent part of the effective interaction $W$ (see e.g. 2, 4, 5, 6, 7, 8] ). Here we investigat effects, associated with long-range spin-spin components of $W$, which involve the spin-up quasiparticle distribution $n_{+}(p)$ and spin-down one $n_{-}(p)$. It is instructive to start such an analysis with a generalization of the Nozieres model [4], supplementing it by long-range spin. spin terms. As a result, one obtains two equations

$$
\begin{aligned}
\xi_{+}(p)= & \xi_{\mathbf{p}}^{0}+\frac{1}{2} f\left(n_{+}(p)+n_{-}(p)\right) \\
& +\frac{1}{2} g\left(n_{+}(p)-n_{-}(p)\right), \\
\xi_{-}(p)= & \xi_{\mathbf{p}}^{0}+\frac{1}{2} f\left(n_{+}(p)+n_{-}(p)\right) \\
& -\frac{1}{2} g\left(n_{+}(p)-n_{-}(p)\right),
\end{aligned}
$$

with a new constant $g$, specifying the long-range spinspin component of the model effective interaction $W$. It is worth noting that in the case of spin fluctuations with the nonzero critical momentum $q_{c} \ll p_{F}$, presumably relevant to two-dimensional liquid He-3, the constants in Eq. (5) are related with each other: $g=-f / 3$.

In conventional Fermi liquids, the spontaneous spin $S$ arises only if strength of the spin-spin interaction obeys the Pomeranchuk condition $|G| \rho(0)>1$, where $\rho(0)=p_{F} M^{*} / \pi^{2}$ is the density of states at the FS in the Landau theory. In the case at issue, the density of states is infinite, so that one can expect the emergence of the spontaneous magnetization at any $g<0$.

To facilitate the solution of the problem, we recast the system (5) to the form

$$
\begin{aligned}
& \xi_{+}=\left(1-\frac{a}{b}\right) \xi_{\mathbf{p}}^{0}+\frac{a}{b} \xi_{-}+\left(b-\frac{a^{2}}{b}\right) n_{-}, \\
& \xi_{-}=\left(1-\frac{a}{b}\right) \xi_{\mathbf{p}}^{0}+\frac{a}{b} \xi_{+}+\left(b-\frac{a^{2}}{b}\right) n_{+},
\end{aligned}
$$

where $a=(f+g) / 2$ and $b=(f-g) / 2$. Let us now draw the plot $\xi_{+}\left(\xi_{-}\right)$, proceeding from the first of Eqs. (6) and treating $p$ as an input parameter. At $\xi_{-}>0$ the function $\xi_{+}\left(\xi_{-}\right)$is given by the straight line $\xi_{+}=(1-a / b) \xi_{\mathbf{p}}^{0}+$ $a \xi_{-} / b$, while at $\xi_{-}<0$ this straight line is slightly shifted: $\xi_{+}=(1-a / b) \xi_{\mathbf{p}}^{0}+\left(b^{2}-a^{2}\right) / b+a \xi_{-} / b$. The drawing of its counterpart $\xi_{-}$from the second of Eqs. (6) yields another couple of straight lines: $\xi_{-}=(1-a / b) \xi_{\mathrm{p}}^{0}+a \xi_{+} / b$ at $\xi_{+}>0$, and $\xi_{-}=(1-a / b) \xi_{\mathbf{p}}^{0}-\left(b^{2}-a^{2}\right) / b+a \xi_{-} / b$ at
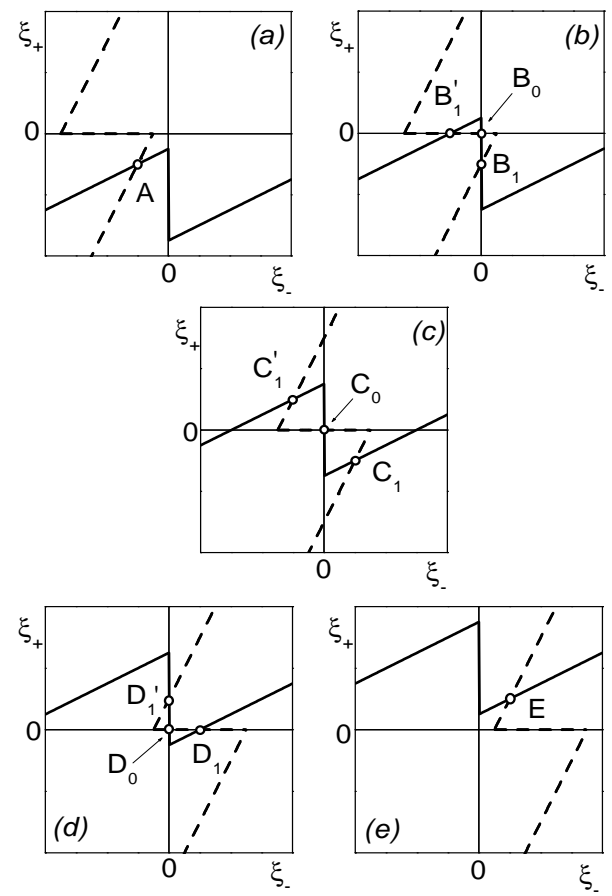

FIG. 2: Graphical illustration of the dynamics of solutions of the set [6] with increasing $\xi_{\mathbf{p}}^{0}$ in the case of $|g|<f$.

$\xi_{+}<0$. As we shall see, both these curves cross each other either at one or at three points, providing several solutions for the spectra $\xi_{ \pm}(p)$.

Some elucidation of the procedure is ensured by Fig. 2, where the evolution of solutions of the set (6) versus the input parameter $\xi_{\mathbf{p}}^{0}$ is shown. The solid line corresponds to the dependence $\xi_{+}\left(\xi_{-}\right)$, given by the first equation of the set ([6), while the dashed one shows the same dependence resulting from the second equation of the set. The intersection points are indicated by letters. Five panels of Fig. 2 show five different cases referring to different $\xi_{\mathbf{p}}^{0}$. In the first case (panel (a)), two zigzag lines have one intersection point $A$, corresponding to the single solution where both $\xi_{+}(p)$ and $\xi_{-}(p)$ are negative. With increasing $\xi_{\mathbf{p}}^{0}$, the intersection point moves to the origin. At a certain value of this variable, depending on the parameter $f$ only, bifurcation emerges, and there appear three intersection points $\left(B_{0}, B_{1}\right.$ and $B_{1}^{\prime}$ on the panel (b)). One of them, $B_{0}$, lies at the origin, while the other two, $B_{1}$ and $B_{1}^{\prime}$, on the $\xi_{+}$and $\xi_{-}$axes, respectively. A similar situation occurs in the panels (c) and (d). The intersection at the origin persists, while the other two crossing points lie inside the quadrants on the panel (c) $\left(C_{1}\right.$ and $\left.C_{1}^{\prime}\right)$ and on the $\xi_{-}$and $\xi_{+}$axes on the panel (d) $\left(D_{1}\right.$ and $\left.D_{1}^{\prime}\right)$. The last (e) panel shows the fifth case with one intersection point at positive values of $\xi_{+}$and $\xi_{-}$.

The chain $A-B_{0}-C_{0}-D_{0}-E$ is associated with the solution (denoted as $\Phi_{0}$ ), for which the sp spectra coincide: $\xi_{+}(p)=\xi_{-}(p)=0$ within the interval $\left[p_{i}, p_{f}\right]$, the length of which is determined by the parameter $f$ only, as if there was no spin-spin interaction at all. The analysis 


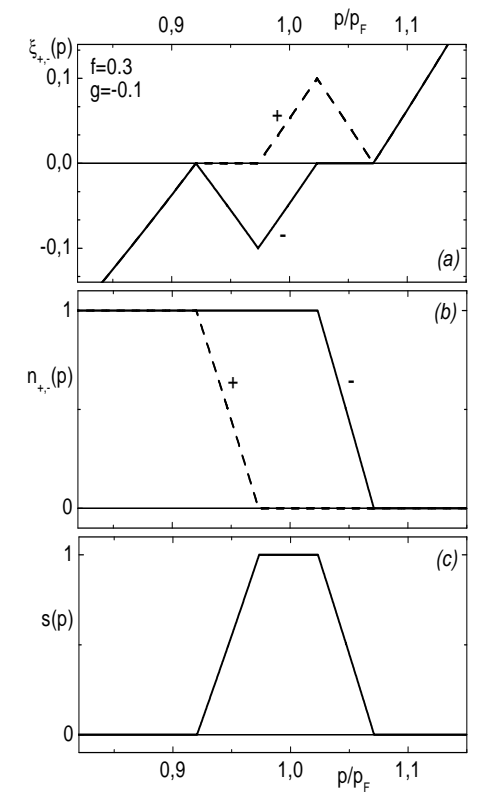

FIG. 3: Single-particle spectra $\xi \pm(p)$ in units of $\varepsilon_{F}^{0}=p_{F}^{2} / 2 M$ (nanel (a)) nccunation numhers $n \perp(n)$ (nanel $(h)$ ) and snon-

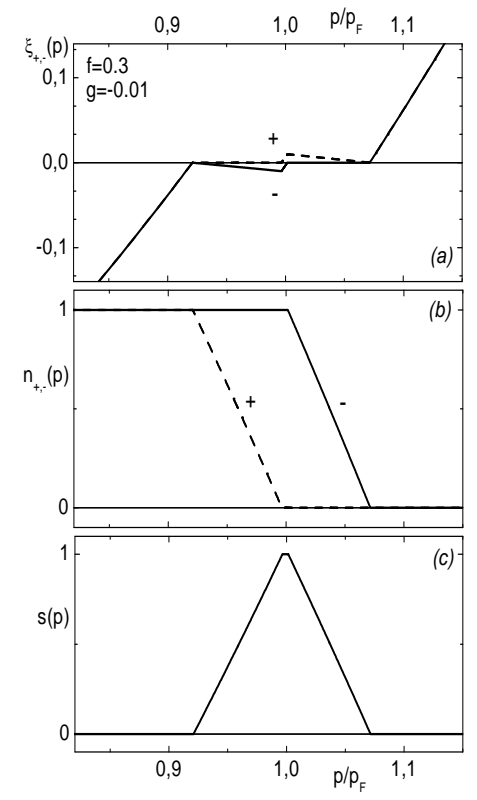

FIG. 4: The same as in Fig. 3 for $f=0.3, g=-0.01$.

shows that, as long as the constant $g$ remains positive, it is the solution $\Phi_{0}$ that has the lowest energy compared with the others.

As for solutions, related to the chains $A-B_{1}-C_{1}-D_{1}-E$ and $A-B_{1}^{\prime}-C_{1}^{\prime}-D_{1}^{\prime}-E \quad\left(\Phi_{1}\right.$ and $\Phi_{1}^{\prime}$, respectively), the situation is different. The solution $\Phi_{1}$, corresponding to the first chain, is shown in Fig. 3. We see that the FC region, the boundaries of which were insensitive to $g$ at any $g>0$, is destroyed: the spectra
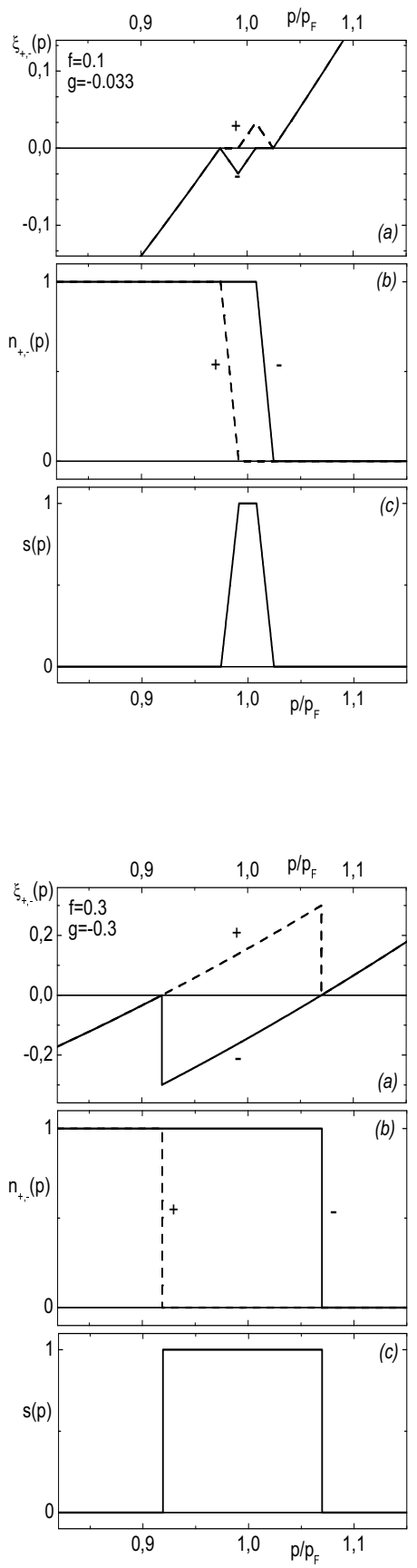

FIG. 6: The same as in Fig. 3 for $f=0.3, g=-0.3$.

$\xi_{+}(p)$ and $\xi_{-}(p)$ repell each other. As the momentum $p$ moves from the lower point, where these spectra simultaneously attain the FS, only the FC plateau at the spectrum $\xi_{+}(p)$ survives, with the energy splitting between the spectra $\xi_{+}(p)$ and $\xi_{-}(p)$ growing linearly with the $p$ increase. Attaining the maximum at the point, where the plateau at $\xi_{+}(p)$ vanishes, this splitting ceases to increase and remains constant until the point, where a new FC plateau emerges at the spectrum $\xi_{-}(p)$, and, finally, both the spectra once again merge at the point, where the FC disappears forever. 

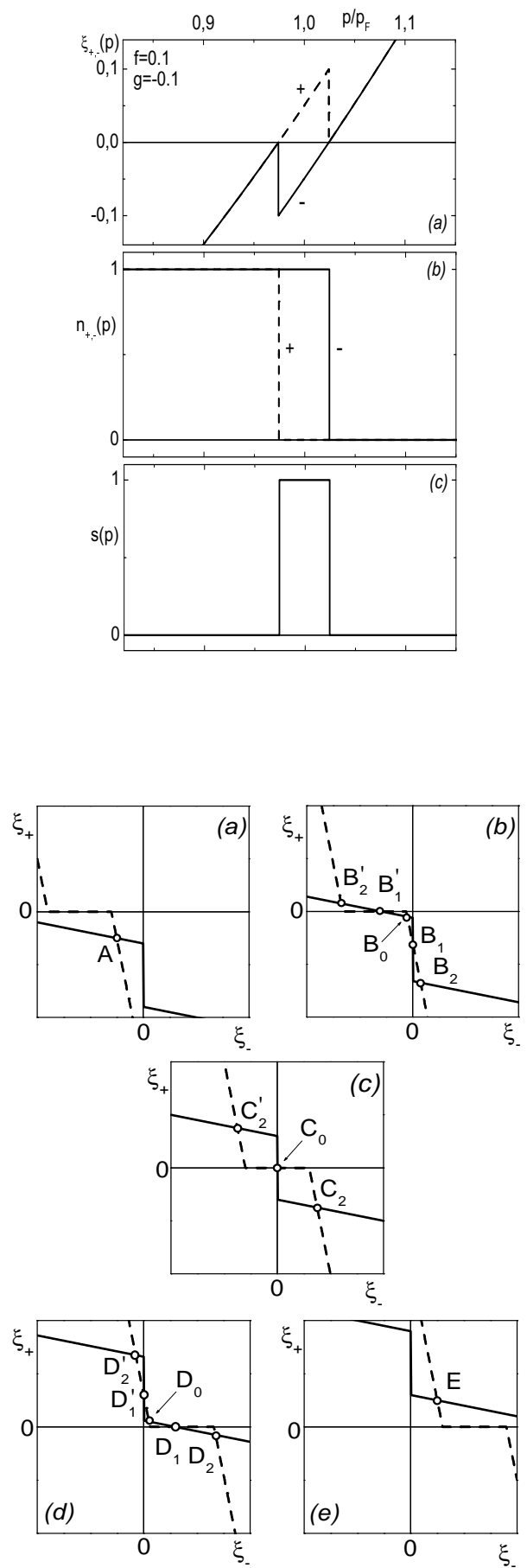

FIG. 8: The same as in Fig. 2 for the case of $|g|>f$.

The second chain refers to the solution $\Phi_{1}^{\prime}$, symmetric to the previous one, with changing \pm by $\mp$ and, thus, corresponds to the opposite sign of the projection of spontaneous spin $S$ on the fixed axis. Since both the directions are equivalent, two solutions with the nonzero $S$ have equal energies. It can be verified that at any $g<0$, these solutions provide the minimum of the ground state energy, while the solution $\Phi_{0}$ with $S=0$, the maximum. Indeed, calculations yield the gain in energy of the so-
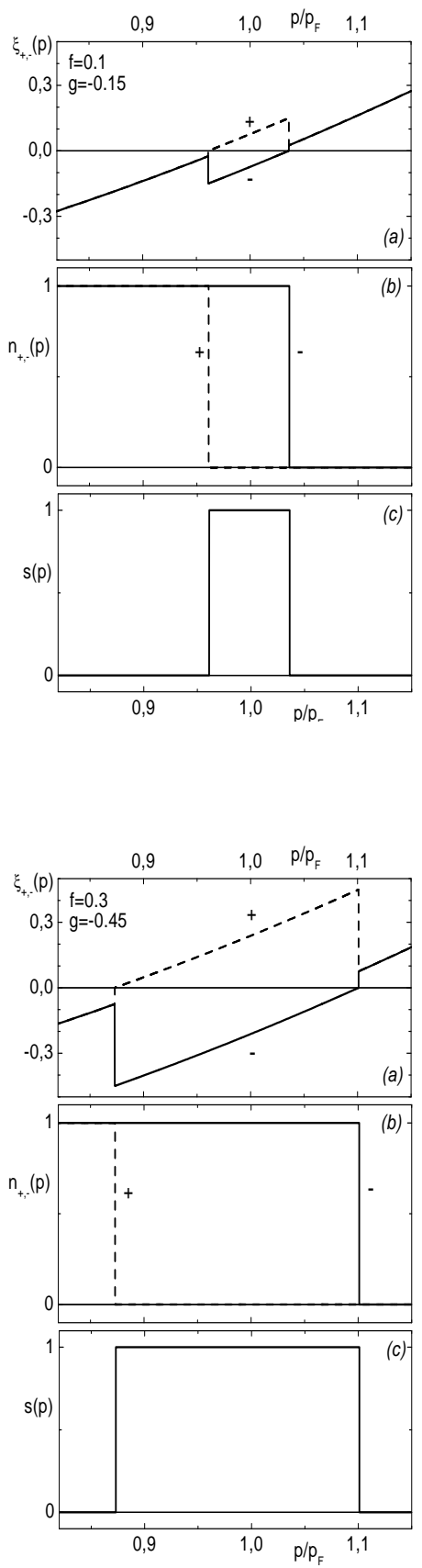

FIG. 10: The same as in Fig. 3 for $f=0.3, g=-0.45$.

lutions $\Phi_{1}$ and $\Phi_{1}^{\prime}$, as compared with the energy of the Landau state $E_{1} / E_{L}-1 \simeq-0.027$, while for the solution $\Phi_{0}$, one obtains $E_{0} / E_{L}-1 \simeq-0.019$.

Thus, the reason for these alterations of the FC is the occurrence of the spontaneous spin

$$
S=\sum_{p} s(p)=\sum_{p}\left(n_{-}(p)-n_{+}(p)\right) .
$$

arising at any $g<0$. For illustration, the spin density $s(p)$ is drawn in panel (c). We infer that the spin density $s(p)$ differs from 0 only in the region $\left[p_{i}, p_{f}\right]$. Therefore 


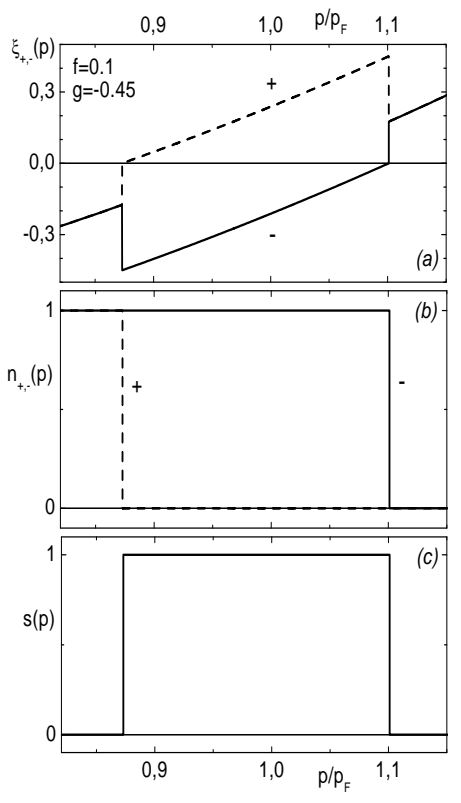

FIG. 11: The same as in Fig. 3 for $f=0.1, g=-0.4$ !

the total spin $S$ depends primarily on the value of rameter $f$. For the parameters $f$ and $g$, given in $\mathrm{Fi}$ one obtains $S / \rho \simeq 0.299$.

For comparison, results for the other two sets of parameters: $f=0.3, g=-0.01$ and $f=0.1, g=-0$ are drawn in Figs. 4 and 5, respectively. The stri feature of these results is that for the given value , 0.3 the magnitude of the spontaneous spin $S$ for $g=$ $-0.01, S / \rho \simeq 0.232$, is of the same order as in the case of $g=-0.1$. The energy gain of the solution with the spontaneous spin, corresponding to $f$ and $g$ for Fig. 4, $E_{1} / E_{L}-1 \simeq-0.019$. Fig. 5 shows that multiplication of both the parameters $f$ and $g$ by the factor $1 / 3$ results in the scaling of the spectra, occupation numbers, and spontaneous spin by the same factor.

The situation drastically changes if the magnitude of the spin-spin force becomes comparable to that of the scalar one. This is seen in Figs. 6 and 7 showing results obtained for the sets $f=0.3, g=-0.3$ and $f=0.1, g=-0.1$. We see that the FC practically disappears, while the Landau state reappears. Now the occupation numbers $n_{+}(p)$ and $n_{-}(p)$ have the Migdal jump from 0 to 1 at the points $p_{i}$ and $p_{f}$, respectively, and the spontaneous spin attains the maximum value, which is equal to the phase volume of the spherical layer between $p_{i}$ and $p_{f}(S / \rho \simeq 0.448$ and $S / \rho \simeq 0.149$, for the parameters of Figs. 6 and 7 , respectively). Fig. 8 illustrates the evolution of solutions for the set (6) versus $\xi_{\mathbf{p}}^{0}$ in the case when the absolute value of the spin-spin constant $g$ is larger than the scalar one $f$. In this case, the set (6) has five solutions: $\Phi_{0}$, corresponding to the chain $A-B_{0}-C_{0}-D_{0}-E ; \Phi_{1}$ and $\Phi_{1}^{\prime}$, corresponding to the chains $A-B_{1}-C_{0}-D_{1}-E$ and $A-B_{1}^{\prime}-C_{0}-D_{1}^{\prime}-E$, respectively; and a new couple
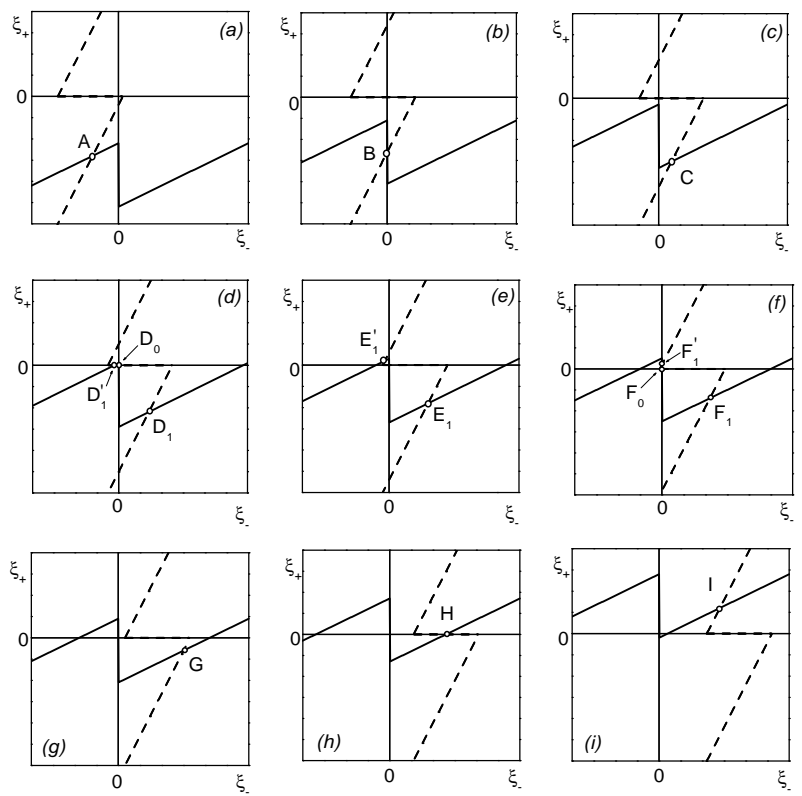

FIG. 12: Graphical illustration of the dynamics of solutions of the set (9) with increasing $\xi_{\mathbf{p}}^{0}$ in the case of $|g|<f, \beta H<$ $|g| / 2$.

of solutions, $\Phi_{2}$ and $\Phi_{2}^{\prime}$, corresponding to the chains $A-B_{2}-C_{2}-D_{2}-E$ and $A-B_{2}^{\prime}-C_{2}^{\prime}-D_{2}^{\prime}-E$. The calculation of the energies yields the solutions $\Phi_{2}$ and $\Phi_{2}^{\prime}$, equal in energy, beating $\Phi_{0}, \Phi_{1}$ and $\Phi_{1}^{\prime}$. The solution $\Phi_{2}$ is drawn in Figs. 9, 10, 11 for three sets of parameters: $f=0.1, g=-0.15 ; f=0.3, g=-0.45 ; f=0.1$, $g=-0.45$. These figures show that in this case the spontaneous spin is defined only by the parameter $g$, and the parameter $f$ defines the magnitudes of the jumps in the spectra $\xi_{+}(p)$ and $\xi_{-}(p)$. It is worth noting that, while the flat portions of the spectra, corresponding to FC, disappear and the Landau state is recovered, the phantom of the FC manifests itself in the emergence of the spontaneous spin.

The above analysis can be generalized to the case of an external magnetic field. In this case, the equations for the sp spectra read

$$
\begin{aligned}
\xi_{+}(p)= & \xi_{\mathbf{p}}^{0}+\frac{1}{2} f\left(n_{+}(p)+n_{-}(p)\right) \\
& +\frac{1}{2} g\left(n_{+}(p)-n_{-}(p)\right)+\beta H, \\
\xi_{-}(p)= & \xi_{\mathbf{p}}^{0}+\frac{1}{2} f\left(n_{+}(p)+n_{-}(p)\right) \\
& -\frac{1}{2} g\left(n_{+}(p)-n_{-}(p)\right)-\beta H,
\end{aligned}
$$

where $H$ is the effective magnetic field acting on the fermion spin and $\beta$ is the magnetic moment of the fermion. Upon rewriting Eqs. (8) to the form convenient 

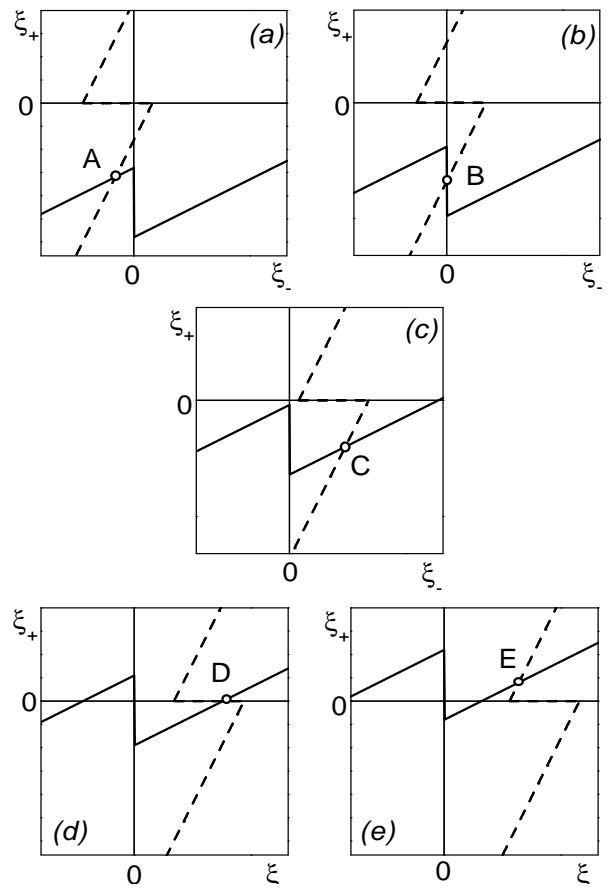

]

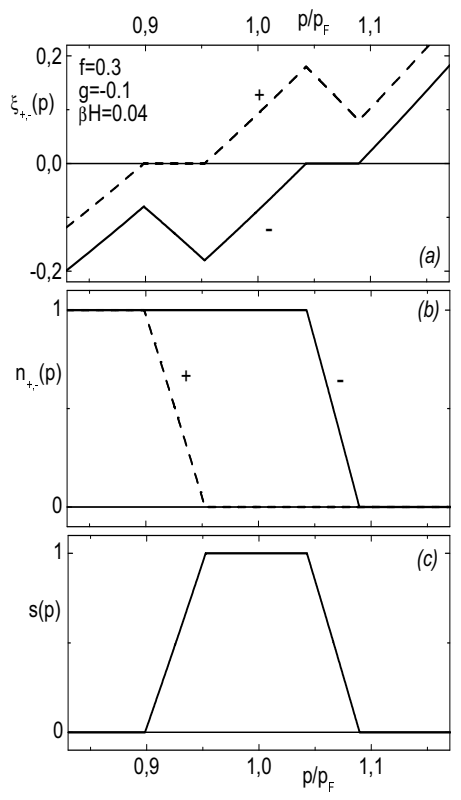

FIG. 14: The same as in Fig. 3 for $f=0.3, g=-0.1$, $\beta H=0.04$.

for the graphical analysis, we obtain

$$
\begin{aligned}
& \xi_{+}=\left(1-\frac{a}{b}\right) \xi_{\mathbf{p}}^{0}+\frac{a}{b} \xi_{-}+\left(b-\frac{a^{2}}{b}\right) n_{-}+\left(1+\frac{a}{b}\right) \beta H, \\
& \xi_{-}=\left(1-\frac{a}{b}\right) \xi_{\mathbf{p}}^{0}+\frac{a}{b} \xi_{+}+\left(b-\frac{a^{2}}{b}\right) n_{+}-\left(1+\frac{a}{b}\right) \beta H .(9)
\end{aligned}
$$

Solutions of Eqs. (9) are represented by the broken lines with the same slopes and jumps as in the case of

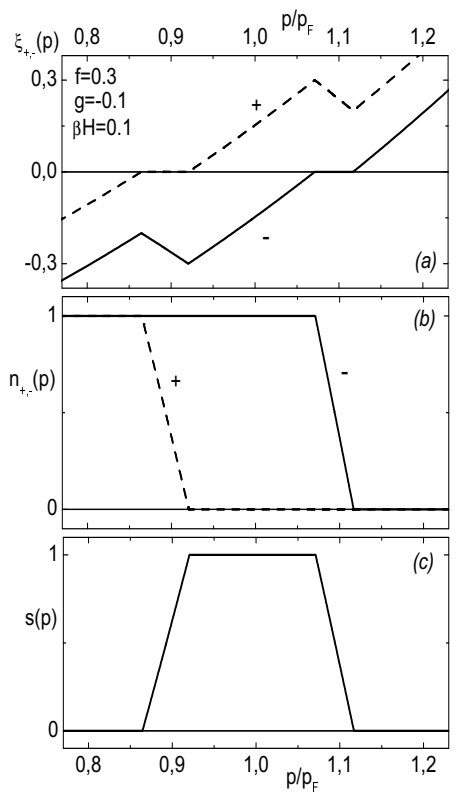

FIG. 15: The same as in Fig. 3 for $f=0.3, g=-0.1$, $\beta H=0.1$.

zero magnetic field but shifted by the value $(1+a / b) \beta H$ along the $\xi_{+}$and $\xi_{-}$axes. For $|g|<f$, two cases can be distinguished. In the case of $\beta H<|g| / 2(\beta H$ is in units of $\varepsilon_{F}^{0}$ ), drawn in Fig. 12, two segments, vertical and horizontal, lying on the axes, intersect each other and the situation with the three intersection points, discussed above, holds. In the case $\beta H>|g| / 2$, the single intersection point of the two lines remains at any $\xi_{0}$ (see Fig. 13). The sp spectra and occupation numbers are shown in Fig. 14 for $f=0.3, g=-0.1, \beta H=0.04$ and in Fig. 15 for the same values of $f$ and $g$ but at $\beta H=0.1$. In both the cases, the magnetic field promotes splitting the sp spectra $\xi_{+}(p)$ and $\xi_{-}(p)$ but does not influence their flat parts.

In conclusion, in the Nozieres-like model of a Fermi system with scalar and spin-dependent long-range forces, it is shown that the fermion condensation, occurring in the vicinity of a phase transition, results in the emergence of the weak magnetization which precedes the ferromagnetic transition.

\section{Acknowledgments}

We thank A. Lichtenstein, G. Kotliar and V. Yakovenko for many valuable discussions.

This research was supported in part by the National Science Foundation under Grants No. PHY99-07949 and No. PHY-0140316, by the McDonnell Center for the Space Sciences at Washington University, and by Grant No. NS-1885.2003.2 from the Russian Ministry of Industry and Science (VAK and MVZ). One of the authors (VAK) thanks the University of California (Santa Bar- 
bara) for the kind hospitality.

[1] S. Doniach and S. Engelsberg, Phys. Rev. Lett. 17, 750, 1966.

[2] V. A. Khodel and V. R. Shaginyan, JETP Lett. 51, 553, 1990.

[3] L. D. Landau, JETP 30, 1058, 1956; 35, 97, 1958.

[4] P. Nozieres, J. Phys. France I 2, 443, 1992.

[5] G. E. Volovik, JETP Lett. 53, 222, 1991.
[6] V. A. Khodel, J. W. Clark and V. R. Shaginyan, Solid St. Comm. 96, 353, 1995.

[7] J. Dukelsky, V. A. Khodel, P. Schuck and V. R. Shaginyan, Z. Phys. B 102, 245, 1997.

[8] M. V. Zverev and M. Baldo, JETP 87, 1129, 1998; J. Phys. Cond. Mat. 11, 2059, 1999. 